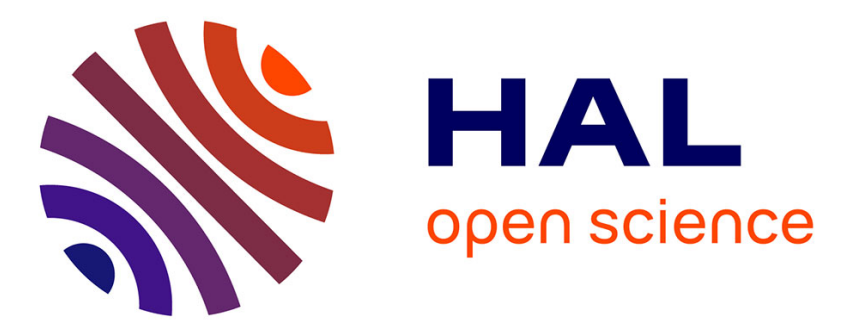

\title{
Optimized Spatial CSMA for VANETs: A Comparative Study using a Simple Stochastic Model and Simulation Results
}

Younes Bouchaala, Paul Mühlethaler, Oyunchimeg Shagdar, Nadjib Achir

\section{- To cite this version:}

Younes Bouchaala, Paul Mühlethaler, Oyunchimeg Shagdar, Nadjib Achir. Optimized Spatial CSMA for VANETs: A Comparative Study using a Simple Stochastic Model and Simulation Results. CCNC 2017. 8-11 january 2017. Las Vegas. , Jan 2017, Las Vegas, United States. hal-01379978

\section{HAL Id: hal-01379978 \\ https://hal.science/hal-01379978}

Submitted on 12 Oct 2016

HAL is a multi-disciplinary open access archive for the deposit and dissemination of scientific research documents, whether they are published or not. The documents may come from teaching and research institutions in France or abroad, or from public or private research centers.

$$
\text { Copyright }
$$

L'archive ouverte pluridisciplinaire HAL, est destinée au dépôt et à la diffusion de documents scientifiques de niveau recherche, publiés ou non, émanant des établissements d'enseignement et de recherche français ou étrangers, des laboratoires publics ou privés. 


\title{
Optimized Spatial CSMA for VANETs: A Comparative Study using a Simple Stochastic Model and Simulation Results
}

\author{
Younes Bouchaala*, Paul Muhlethaler ${ }^{\dagger}$, Oyunchimeg Shagdar*, and Nadjib Achir ${ }^{\ddagger}$ \\ *Institut VEDECOM, 77, rue des Chantiers, 78000 Versailles, France \\ Emails: \{younes.bouchaala, oyunchimeg.shagdar\}@ vedecom.fr \\ ${ }^{\dagger}$ INRIA EVA, Centre de Recherche de Paris, 2 Rue Simone, IFF CS 42112, 75589 Paris Cedex 12 \\ Email: \{paul.muhlethaler\}@inria.fr \\ $\ddagger$ Universite Paris 13, Sorbonne Paris Cite - L2TI, 99 Avenue J-B Clément, 93430 Villetaneuse, France \\ Email: nadjib.achir@univ-paris13.fr
}

\begin{abstract}
The high densities of network nodes has made spatial reuse an essential characteristic of modern wireless networks. In this paper, we evaluate the maximum throughput of Carrier Sense Multiple Access (CSMA) for Vehicular Ad-hoc Networks (VANETs) when spatial reuse is taken into account. We begin our study by extending a simple stochastic model in order to fit a VANET pattern and to obtain the spatial density of throughput in terms of the main network parameters. This model uses a Matern selection process with a random pattern of nodes distributed as a Poisson Point Process (PPP). Each node of the process receives a random mark and the nodes that have the smallest mark in their neighborhood are elected for transmission. We study both 1D and 2D network cases with an SIR (Signal over Interference Ratio) model. In order to verify the correctness of the model, extensive simulations are carried out using two simulation platforms: the network simulator, ns-3, and a simulator which is dedicated to CSMA systems. Fairly good matching between the results of the model and those obtained from simulators are observed, confirming the reliability of the theoretical model. Although the results did not perfectly match due to the number of assumptions made for the model, the results obtained nonetheless show the potential for a significant improvement in the overall throughput for VANETs and similar distributed networks.
\end{abstract}

Keywords-VANETs, CSMA, spatial performance, stochastic geometry.

\section{INTRODUCTION}

Until recently, most common Wireless Local Area Networks (WLANs) had one access point which was a gateway to the Internet. These wireless networks, which followed the IEEE 802.11 standard, could only support one transmission at a time as all the nodes were within the same limited geographic area. Moreover, all the nodes were competing for access to the same gateway. Today, recent wireless networks are much larger and more scattered and their transmission patterns are more complex. For instance, Wireless Sensor Networks (WSNs), which are deployed in various domains such as industrial plants, fire detection and prevention, and precision agriculture, show a complex pattern which supports simultaneous transmission and a high delivery rate. This is also the case for Mobile Ad-hoc NETworks (MANETs) and Vehicular Adhoc NETworks (VANETs) whose topology is generally linear. In these networks, transmission can be multihop, particularly when the distance between the transmitter and the receiver nodes is large. This leads to a situation where simultaneous transmissions are likely to happen, especially when spatial reuse is possible. VANETs are characterized by extended linear topologies and a high density of communicating nodes. This makes spatial reuse a vital feature for VANETs. Nowadays, most WLANs are based on the well known IEEE 802.11 MAC protocol which is a random access scheme using a Carrier Sense technique (CSMA). Somewhat surprisingly, in VANETs, the access protocol follows the IEEE 802.11p standard which is also a CSMA-based protocol. This means that both WLANs and VANETs are using a similar access technique which is inadequate given the differences between these two network types in terms of requirements. The same can be said for WSNs that commonly adopt the IEEE 802.15.4 standard which is once again based on a CSMA protocol.

While CSMA has been widely studied over the last decade, spatial CSMA has received very little attention due to the characteristics of WLANs, and it is clear that greater attention should be paid to spatial reuse for VANETs, WSNs, and other large distributed wireless networks. In particular, spatial reuse properties require further analysis so that enhance networking performance can be achieved. Analyzing spatial CSMA is considerably more challenging than evaluating spatial Aloha [1] because simultaneous transmission patterns in spatial CSMA are much harder to model due to their increased complexity.

The remainder of this paper is organized as follows: Section II briefly reviews related work; Section III describes the model proposed to study CSMA and develops the corresponding analytical model. The results of the model evaluating the influence of the parameters are reported in Section IV. Finally, Section V concludes the paper.

\section{RELATED WORK}

The first studies of CSMA were carried out in the 1970s and many papers followed Kleinrock [2] in analyzing an ideal CSMA where all the nodes were never beyond carrier sense range of each other. Although the carrier sense access technique was accurately modeled in [2], analyses of the backoff techniques involved were lacking in detail. In the early 2000s, Bianchi's model [3] represented a major advance in terms of saturation throughput computation. However, the CSMA backoff technique described considered only a one-hop 
network, and so the possibility of addressing spatial reuse was excluded.

The first studies which attempted to take spatial reuse into account appeared in the 1980s and concerned only the Aloha protocol [4] [1]. A model for slotted Aloha was later introduced in 1988 by Ghez, Verdu and Schwartz . Their quantitative model was able to capture the situation where several receptions were possible at different places in the network. In [1] the performance of a network based on this same model was more accurately evaluated. In particular, the capture probability and the density of successful transmissions were computed when the distance between the source and the destination was known. These evaluations were possible due to the complete and stateless randomization of the transmitting nodes in Aloha networks. The CSMA interference issue was raised in [5] for a linear network of randomly positioned vehicles, however, the study only considered the nearest interferer case.

The pattern of simultaneous transmissions in CSMA was first evaluated in [6] using the Matern selection process [7]. An other similar process was used in [8] in order to evaluate interferences in CSMA, but, the study did not assess the overall network throughput. The model initially developed in [6] and subsequently enhanced in [9], is once again being extended in this current work.

While the effect of the carrier sense detection threshold in CSMA protocols has been studied in [10],[11], the spatial effect of this threshold was not taken into account. Rather, these studies focused more on the capture probability when all nodes are within the same one-hop range.

\section{SYSTEM MODEL}

In this paper, we consider nodes randomly deployed according to a Poisson-Point-Process (PPP) $\Phi$ expanded over a 2D plane $\left(\mathcal{S}=\mathbb{R}^{2}\right)$, or along a $1 \mathrm{D}$ infinite line $\mathcal{S}=\mathbb{R}$. The $2 \mathrm{D}$ model is primarily intended for Mobile Ad-hoc NETworks (MANETs) or Wireless Sensor Networks (WSNs), whereas the 1D model is better suited to Vehicular Ad-hoc NETworks (VANETs). We denote by $\lambda$ the intensity of the PPP.

We assume a random fading and a power-law in a distance decay $1 / r^{\beta}$ where $\beta$ varies between 3 and 6 depending on the propagation conditions. We take the fading to be Raleigh i.e. exponentially distributed with parameter $\mu$ and thus a mean of $1 / \mu$. Hence, the signal received when the transmitter and the receiver are at distance $r$ from each other is $F / l(r)^{1}$ with $l(r)=r^{\beta}$. We also use the well-accepted $\operatorname{SIR}^{2}$ (Signal over Interference Ratio) with a capture threshold $T$.

In order to emulate the CSMA selection process, we use a Matern selection process. The principle consists in attributing a random mark $m_{i}$ to each node $X_{i} \in \Phi$. We denote by $F_{i, j}$ the fading for a transmission between $X_{i}$ and $X_{j}$. The Matern process essentially selects the points $X_{i}$ with the smallest random marks $m_{i}$ in their neighborhood. In order to define the neighborhood of a point $X_{i}$, we also introduce the carrier sense threshold $P_{c s}$. We define the neighborhood of $X_{i}$ as being $\mathcal{V}\left(X_{i}\right)=\left\{X_{j} \in X_{i} F_{i, j} / l\left(\left|X_{i}-X_{j}\right|\right)>P_{c s}\right\}$. A

\footnotetext{
${ }^{1}$ The power received $P=\frac{P_{0} F}{l(r)}$ and we set $P_{0}=1$

${ }^{2}$ We omit the thermal noise but it could be easily added, as is explained below. An even more realistic model than the SIR based on a graded SIR model using Shannon's law is possible in our framework though with an increased computational cost. This will be discussed below.
}

node, $X_{i}$ for instance, will be selected by the Matern selection process if and only if $\forall X_{j} \in \mathcal{V}\left(X_{i}\right) m_{i}<m_{j}$, i.e $X_{i}$ has the smallest mark $m_{i}$ in its neighborhood.

In a real CSMA system, once a node has been eliminated by the selection process, the eliminated node is no longer allowed to eliminate other nodes. However, the Matern selection process may lead to an over-elimination of nodes, as illustrated in Figure III.1. Following the Matern selection process, node $i$ correctly eliminates node $o$, even so, node $o$ is still able to eliminate node $p$. In contrast, in a real CSMA system, once node $i$ has eliminated node $o$, node $o$ can no longer eliminate any other neighbor.

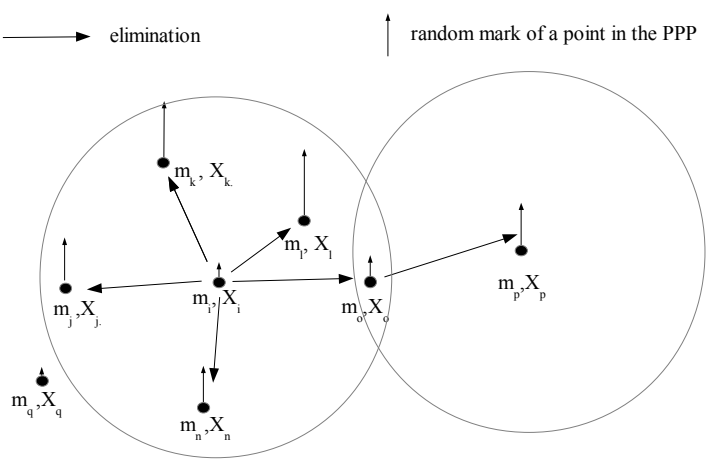

Fig. III.1. Matern CSMA selection process and an example of overelimination.

We note the medium access indicator of node $X_{i} e_{i}=$ $\mathbb{I}\left(\forall X_{j} \in \mathcal{V}\left(X_{i}\right) \quad m_{i}<m_{j}\right)$

Proposition III.1. The mean number of neighbors of a node is:

$$
\mathcal{N}=\lambda \int_{\mathcal{S}} P\left\{F \geqslant P_{c s} l(|x|)\right\} d x .
$$

In a $1 D$ network we have :

$$
\mathcal{N}=\frac{\lambda \Gamma(1 / \beta)}{\beta\left(P_{c s} \mu\right)^{1 / \beta}} .
$$

In a $2 D$ network we have :

$$
\mathcal{N}=\frac{2 \pi \lambda \Gamma(2 / \beta)}{\beta\left(P_{c s} \mu\right)^{2 / \beta}} .
$$

This result is straightforward. Let $F_{j}^{0}$ be the fading between the transmitting node at the origin $X_{i}$ and the receiving node $X_{j}$. This is just the application of Slivnyak's theorem and Campbell's formula, see [12], [9]

$$
\begin{aligned}
\mathcal{N} & = & E^{0}\left[\sum_{X_{j} \in \phi} \mathbb{I}\left(F_{j}^{0} l\left(\left|X_{j}-X_{i}\right|\right) \geqslant P_{c s}\right]\right. \\
& = & \lambda \int_{\mathcal{S}} P\left\{F \geqslant P_{c s} l(|x|)\right\} d x
\end{aligned}
$$

An immediate computation yields the explicit value of $\mathcal{N}$ in the $1 \mathrm{D}$ and $2 \mathrm{D}$ cases.

Proposition III.2. The probability $p$ that a given node $X_{0}$ transmits i.e. $e_{0}=1$ is:

$$
p=\mathbf{E}^{0}\left[e_{0}\right]=\frac{1-e^{-\mathcal{N}}}{\mathcal{N}} .
$$


Proof: We compute the probability of a given node at the origin with the mark $m=t$ being allowed to transmit. Deconditioning on $t$ provides the result, see [9] for details.

If $p$ is close to 1 , then the carrier sense threshold imposes no restriction on transmission. On the other hand, if $p$ is close to 0 , then the carrier sense threshold imposes a severe restriction on transmission.

Proposition III.3. The probability that $X_{0}$ transmits given that there is another node $X_{j} \in \Phi$ at distance $r$ is $p_{r}$ with

$$
p_{r}=p-e^{-P_{c s} \mu l(r)}\left(\frac{1-e^{-\mathcal{N}}}{\mathcal{N}^{2}}-\frac{e^{-\mathcal{N}}}{\mathcal{N}}\right)
$$

Proof: The proof is the same as that of Proposition III.2.

Proposition III.4. Let us suppose that $X_{1}$ and $X_{2}$ are two points in $\Phi$ such that $\left|X_{1}-X_{2}\right|=r$. We suppose that node $X_{2}$ is retained by the selection process. The probability that $X_{1}$ is also retained is:

$$
h(r)=\frac{\frac{2}{b(r)-\mathcal{N}}\left(\frac{1-e^{-\mathcal{N}}}{\mathcal{N}}-\frac{1-e^{-b(r)}}{b(r)}\right)\left(1-e^{-P_{c s} \mu l(r)}\right)}{\frac{1-e^{-\mathcal{N}}}{\mathcal{N}}-e^{-P_{c s} \mu l(r)}\left(\frac{1-e^{-\mathcal{N}}}{\mathcal{N}^{2}}-\frac{e^{-\mathcal{N}}}{\mathcal{N}}\right)}
$$

with

$$
b(r)=2 \mathcal{N}-\lambda \int_{\mathcal{S}} e^{-P_{c s} \mu(l(|x|)+l(|r-x|)} d x .
$$

In a $1 D$ network, we have:

$$
b(r)=2 \mathcal{N}-\lambda \int_{-\infty}^{\infty} e^{-P_{c s} \mu(l(\tau)+l(|r-\tau|))} d \tau
$$

In a $2 D$ network, we have:

$b(r)=2 \mathcal{N}-\lambda \int_{0}^{\infty} \int_{0}^{2 \pi} e^{-P_{c s} \mu\left(l(\tau)+l\left(\sqrt{\tau^{2}+r^{2}-2 r \tau \cos (\theta)}\right)\right)} d \tau d \theta$.

Proof: The proof can be found in [9]

Proposition III.5. Given the transmission of a packet, we denote by $p_{c}\left(r, P_{c s}\right)$ the probability of this packet being successfully received at distance $r$ in a CSMA system (modeled by a Matern selection process with a carrier sense threshold $\left.P_{c s}\right)$ and with a capture threshold $T$. We have:

$$
p_{c}\left(r, P_{c s}\right) \simeq \exp \left(-\lambda \int_{\mathcal{S}} \frac{h(|x|)}{1+\frac{l(|x-r|)}{T l(r)}} d x\right)
$$

In a $1 D$ network, we have:

$$
p_{c}\left(r, P_{c s}\right) \simeq \exp \left(-\lambda \int_{-\infty}^{\infty} \frac{h(\tau)}{1+\frac{l(|r-\tau|)}{T l(r)}} d \tau\right)
$$

In a $2 D$ network, we have:

$p_{c}\left(r, P_{c s}\right) \simeq \exp \left(-\lambda \int_{0}^{\infty} \int_{0}^{2 \pi} \frac{\tau h(\tau)}{1+\frac{l\left(\sqrt{\tau^{2}+r^{2}-2 r \tau \cos (\theta)}\right)}{T l(r)}} d \tau d \theta\right)$

Proof: Assuming a packet is transmitted, $p_{c}\left(r, P_{c s}\right)$ denotes the probability of this packet being successfully received at distance $r$ in a CSMA system using a Matern selection process with carrier sense threshold $P_{c s}$ and with a capture threshold $T$.

The idea is to consider a transmitter at the origin and to evaluate the probability of successful reception by a receiver located at distance $r$. We condition the reception of a packet by the presence of another transmitting node at distance $\tau$. According to proposition III.4, the density of such nodes is $\lambda h(\tau)$. We obtain the result by integrating on $\tau$. The details of the proof can be found in [9].

It is easy to add a thermal noise $W$ to the model. The expression of $p_{c}\left(r, P_{c s}\right)$ must then be multiplied by $\mathcal{L}_{W}(\mu T l(r))$ where $\mathcal{L}_{W}($.$) is the Laplace Transform of the noise.$

In a more advanced model, using Shannon's law we have the average spatial rate $\tau$

$$
\mathbf{E}(\log (1+S I R))=\int_{0}^{\infty} p_{c}\left(r, P_{c s}, e^{t}-1\right) d t
$$

with $p_{c}\left(r, P_{c s}, x\right)=p_{c}\left(r, P_{c s}\right)$ where $T$ is substituted by $x$, see [9]. Although more complicated, such an approach seems computationally achievable, and will form the subject of a more extensive study of spatial CSMA.

Proposition III.6. The spatial density of successful transmissions is thus:

$$
\lambda p p_{c}\left(r, P_{c s}\right)
$$

There are $1 D$ and $2 D$ versions of this spatial density and the value of $p$ and $p_{c}\left(r, P_{c s}\right)$ are chosen accordingly.

Proof: Proposition III.6 is just the exploitation of propositions III.2 and III.5.

We now compute the spatial density of successful transmissions when the packets are sent to the closest neighbor. We have the following result.

Proposition III.7. The spatial density of successful transmissions for a $1 D$ network is:

$$
\lambda^{2} p \int_{0}^{\infty} p_{c}\left(x, P_{c s}\right) e^{-\lambda x} d x
$$

and for a $2 D$ network the density of successful transmissions is:

$$
2 \lambda^{2} \pi p \int_{0}^{\infty} x p_{c}\left(x, P_{c s}\right) e^{-\lambda \pi x^{2}} d x
$$

Proof: Proposition III.7 is just the exploitation of proposition III.6 and the spatial density of a neighbor in 1D and 2D networks.

\section{RESULTS OF THE MODEL}

In this section, we compare the results obtained by the mathematical model with those derived by simulations. In the evaluations, the nodes (vehicles) are distributed following the Poisson Point Process in 1D and 2D geographical areas. Then, we study the transmissions for pairs of source-destination nodes at distance $r$ which are $1 / \lambda$ and $1 / 2 \sqrt{\lambda}$ for $1 \mathrm{D}$ and $2 \mathrm{D}$ networks respectively. $r$ can be seen as a typical distance in these networks since it is the average distance between a node and its closest neighbor. We also study the successful transmission rate to the closest neighbor for each transmitter. 
For the simulations we use two different tools. The first one is the ns-3 simulator which implements a large number of networking modules including the IEEE 802.11p, which is the key module for the current study. The second tool is a simulator that implements a simple IEEE 802.11 CSMA/CA protocol using the $\mathrm{C}$ programming language. In contrast to ns-3, thanks to its simple architecture the simulation run time is extremely short in our dedicated simulator. Since the mathematical model is made for the saturated scenario, the packet generation intervals in the simulations are set to very short values in such a way that all the nodes always have a packet to send. Moreover, the modulation rate is set to $6 \mathrm{Mbits} / \mathrm{s}$, the packet size is set to 4000 bits, and the back-off window is 31 slots. To compare the results obtained by simulations to those provided by the model, we also compute the density of successful transmissions for the simulations, taking into account the number of successfully received packets, the duration of the packet, the simulation duration and the size of the network.

For the carrier sense threshold parameter, we use the usual power measurement unit i.e. decibels $(\mathrm{dB})$. If, for instance, the carrier sense threshold is $x d B$, the $P_{c s}$ used in the analytical model will be $10^{-\frac{x}{10}}$. Moreover, we set the value of signal decay in both the analytical model and the dedicated IEEE 802.11 CSMA/CA simulator to fit the default value of signal decay configured in ns-3 which is 2 .

\section{A. Density of successful transmissions versus the carrier sense threshold $P_{c s}$}

We start our work by studying the case of $1 \mathrm{D}$ networks. The simulation scenario consists in having a random distribution of nodes, whose density $\lambda=0.05$, in a $1 \mathrm{D}$ network of 1000 meters of length. This means that the average inter-car (nodes) distance is equal to 20 meters, and thus, the positions of the nodes are $x$ randomly selected between 0 and 1000 meters. To fit the simulation configuration to the conditions of the model, we have set two nodes at predefined positions: the sender at position $(\mathrm{x}=490, \mathrm{y}=0)$ and the receiver at position $(\mathrm{x}=510$, $\mathrm{y}=0$ ), and randomly distributed the rest of the nodes (see Figure IV.1). The main purpose of this scenario is to study the successful transmission rate between two nodes positioned at a mean distance (which depends on node density) from each other. Figure IV.3 shows a comparison of the density of successful transmissions obtained by both the analytical model and the ns-3 simulator ${ }^{3}$. The comparison shows a fairly good matching. In our study, we observed that the condition for the successful reception of a packet in ns-3 is implemented in a much more complex way than the simple signal-overinterference-and-noise ratio model. The successful reception of a packet in ns-3 is conditioned by a random variable of the signal over the interference ratio, see [13]. We observe that the successful reception conditions in ns-3 roughly correspond to the analytical model, with a capture threshold $\mathrm{T}$ of between 5 and 10. Figure IV.4 shows a comparison of the density of successful transmissions obtained by both the analytical model and our dedicated IEEE 802.11 CSMA/CA simulator. This time, we observe a better overall matching between the two approaches. This is probably due to the fact that the settings of our CSMA/CA simulator are closer to the analytical model than those of the ns-3 simulator.

\footnotetext{
${ }^{3}$ The error bars provide the $95 \%$ confindence interval in all the figures presented
}

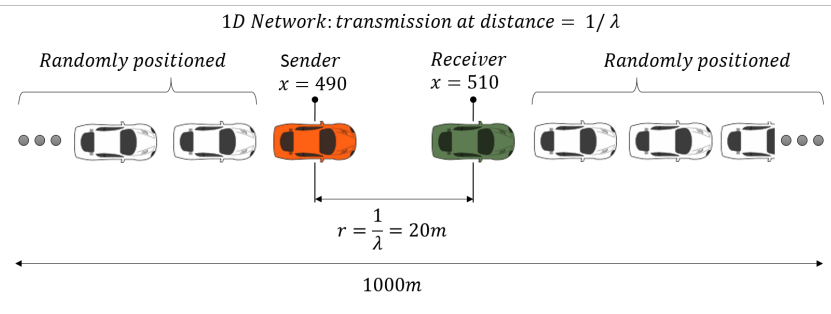

Fig. IV.1. 1D Network: transmission at distance $1 / \lambda$.

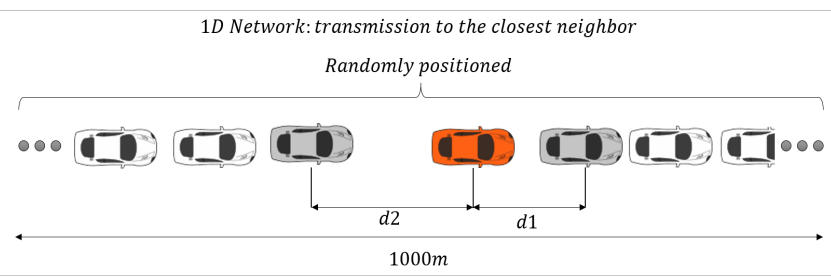

Fig. IV.2. 1D Network, transmission to the closest neighbor.

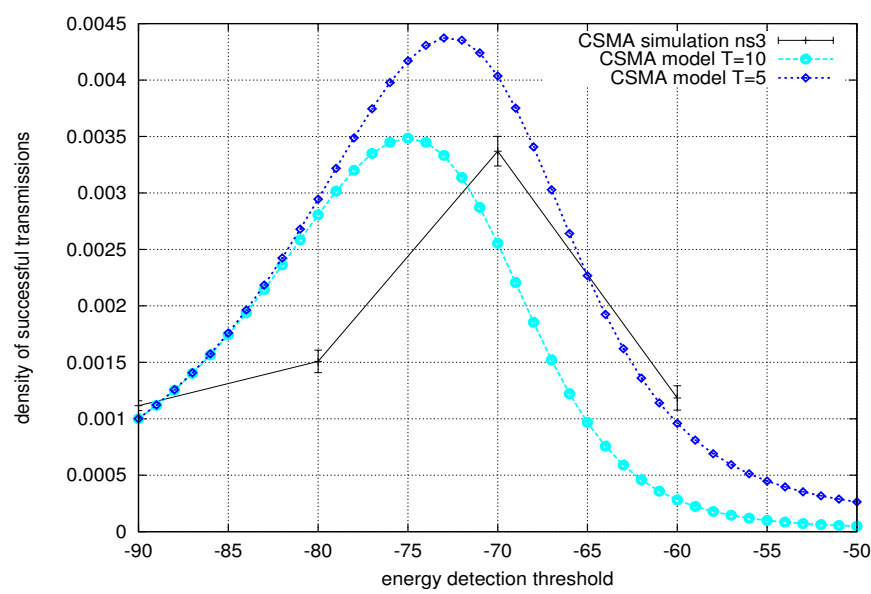

Fig. IV.3. Density of successful transmissions versus carrier sense threshold ns3 simulation and analytical model for $T=5$ and $T=10, \beta=2$ in a $1 D$ network for a transmission at distance $1 / \lambda=20 \mathrm{~m}$.

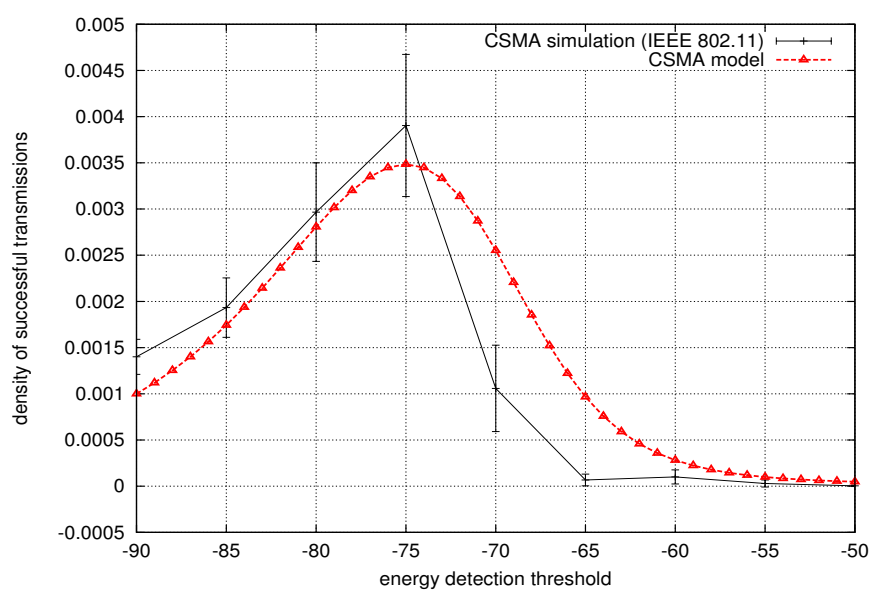

Fig. IV.4. Density of successful transmissions versus carrier sense threshold for the IEEE 802.11 simulation and the analytical model $T=10, \beta=2,1 \mathrm{D}$ network for a transmission at distance $1 / \lambda=20 \mathrm{~m}$. 
We then study the transmission from each node to its closest neighbor as shown in Figure IV.2. We count the successful transmissions from each node to its closest neighbor and compute the spatial density of these successful transmissions. Figure IV.5 illustrates the comparison between the analytical model and ns-3 simulation results. The matching is fair, and the density of successful transmissions increases with the carrier sense threshold as predicted by the analytical model. The increase is comparable in both the simulation and the model. In Figure IV.6 the same comparison is made between the analytical model and the IEEE 802.11 CSMA/CA simulator. We observe a reasonable matching between both approaches and the matching is better for larger values of the carrier sense threshold than in the previous comparison in Figure IV.5. This is probably due to the way the carrier sense threshold is implemented in ns-3. It does not actually take into account the reception of packets below the energy detection threshold. This point will be further detailed below.

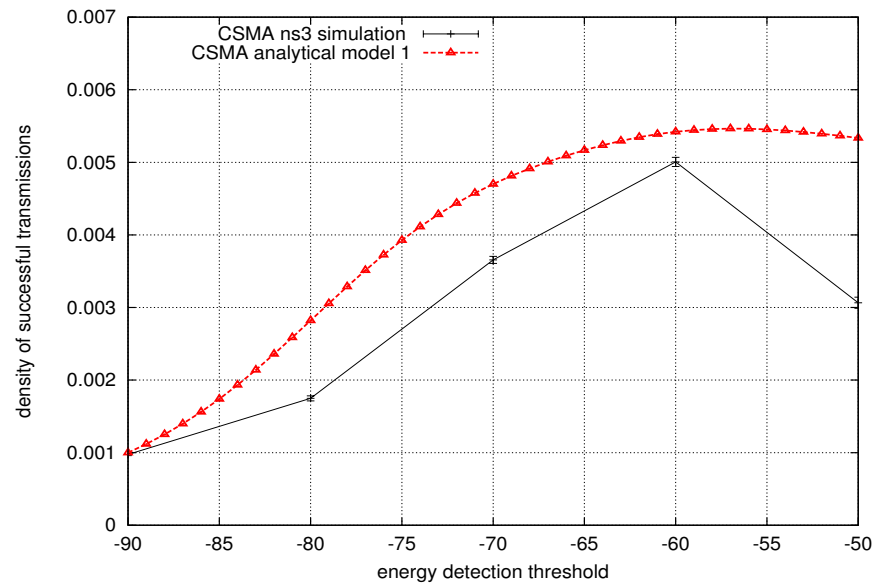

Fig. IV.5. Density of successful transmissions versus carrier sense threshold for the IEEE 802.11 simulation and the analytical model $T=10, \beta=2,1 \mathrm{D}$ network for a transmission to the closest neighbor.

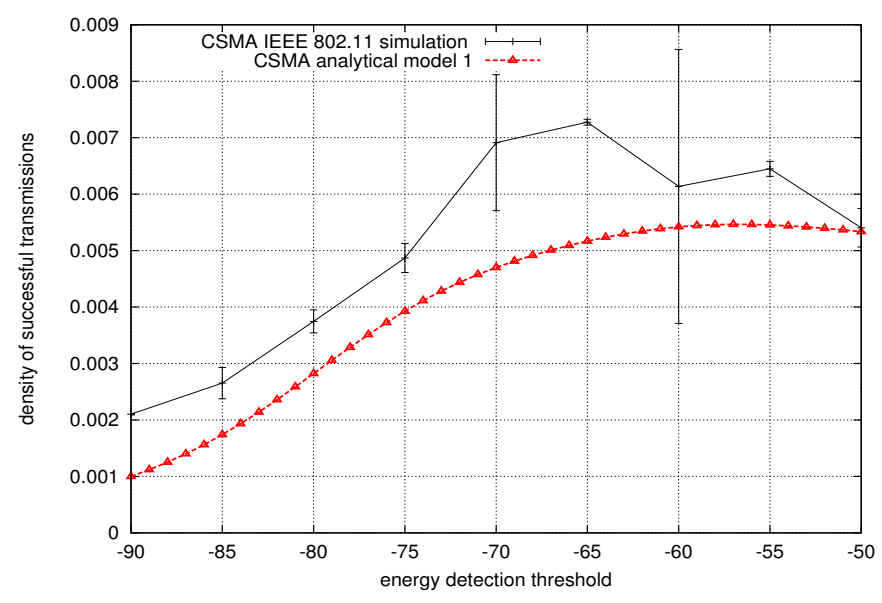

Fig. IV.6. Density of successful transmissions versus carrier sense threshold for the IEEE 802.11 simulation and the analytical model $T=10, \beta=2,1 \mathrm{D}$ network for a transmission to the closest neighbor.

\section{B. 2D networks: comparing simulations and the model}

Here, we compare the results obtained by the analytical model to those obtained by simulation for $2 \mathrm{D}$ networks. We set $\lambda=0.0025$ and $\beta=3$. The mean distance from one node to its closest neighbor is $1 / 2 \sqrt{\lambda}=10 \mathrm{~m}$. The scenarios of the simulations are presented in Figures IV.9 and IV.10. For this study, we only consider the results obtained by our IEEE 802.11 simulator. The results of the comparison are shown in Figures IV.7 and IV.8. The matching is fair, in particular the range of the increase in the density of successful transmissions is well estimated by the model, but the value of the carrier sense threshold for which the density of successful transmissions is maximized is not so well estimated.

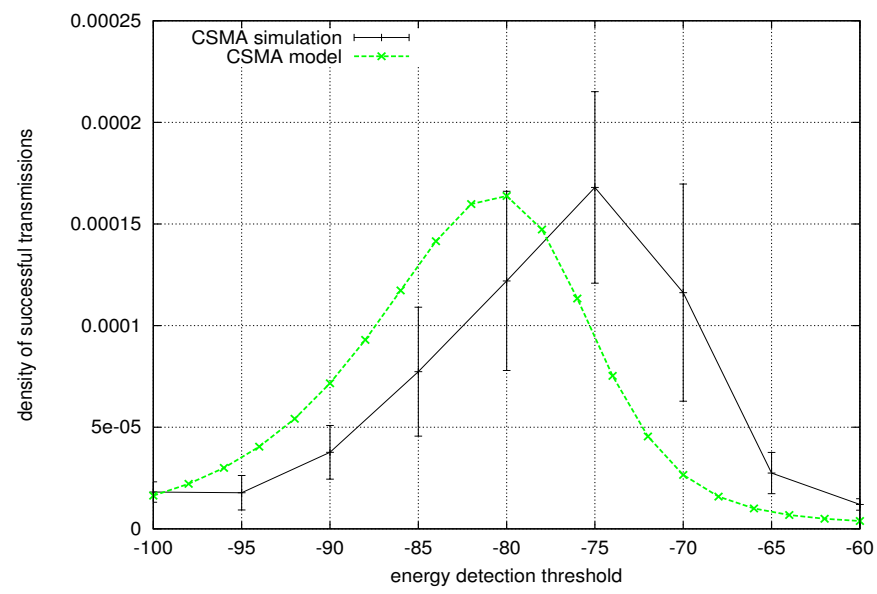

Fig. IV.7. Density of successful transmissions versus carrier sense threshold for the IEEE 802.11 simulation and the analytical model $T=10, \beta=3,2 \mathrm{D}$ network for a transmission at distance $1 / 2 \sqrt{\lambda}=10 \mathrm{~m}$.

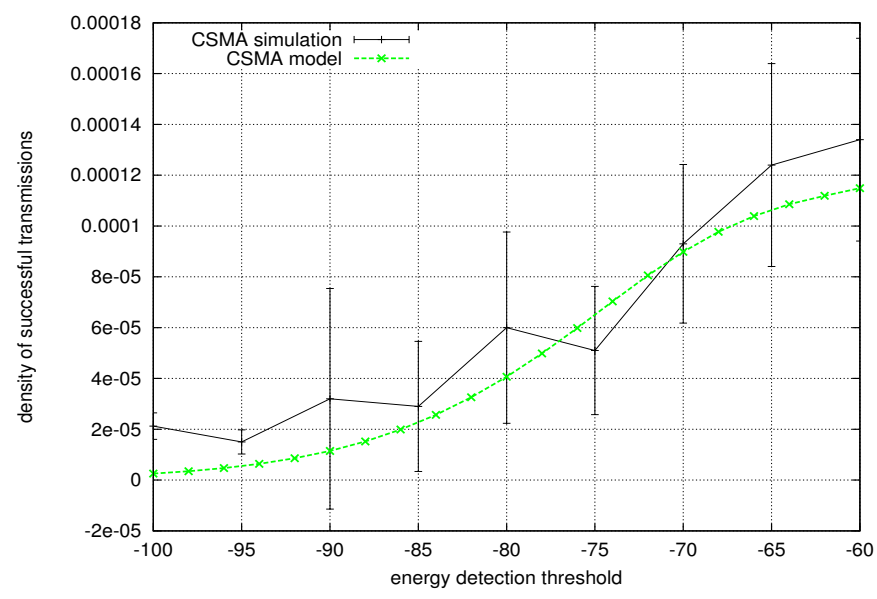

Fig. IV.8. Density of successful transmissions versus carrier sense threshold for the IEEE 802.11 simulation and the analytical model $T=10, \beta=3,2 \mathrm{D}$ network for a transmission to the closest neighbor.

\section{Analysis of the results obtained}

In this section we attempt to explain the reasons for the small discrepancies present in some of our comparison results. We believe that the reasons are:

- the model uses an imperfect modeling of the CSMA selection process (see Section III), and moreover the 


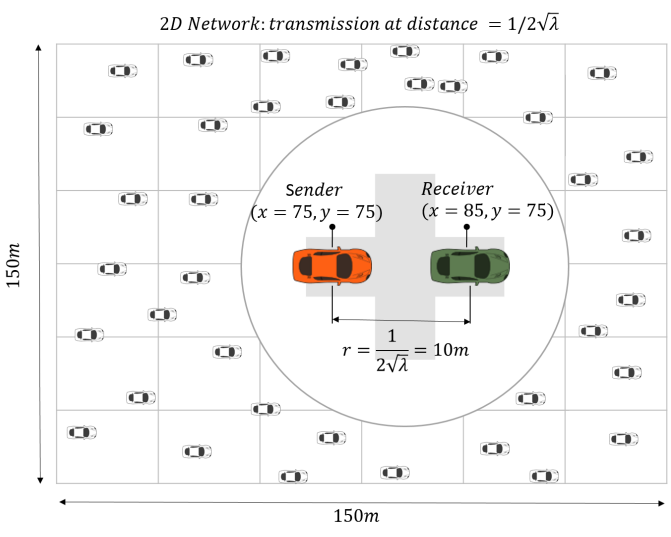

Fig. IV.9. 2D Network, transmission at the distance $1 /(2 \sqrt{\lambda})$.

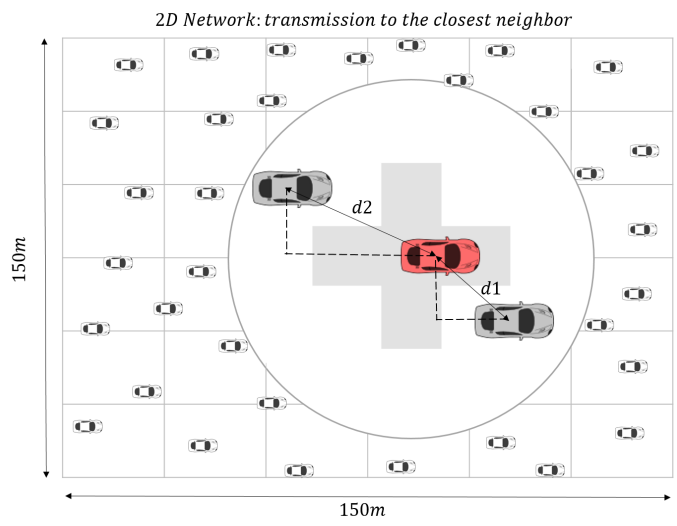

Fig. IV.10. 2D Network, transmission to the closest neighbor.

scheme used in the model is slotted whereas the real protocol is non-slotted,

- the model does not take into account the time spent by the protocol in the back-off state and thus the density of successful transmissions might well be overestimated,

- the model completely neglects the real collisions which occur when different nodes start transmitting their packets at nearly the same time,

- ns-3 uses two thresholds : the energy detection threshold and the carrier-sense threshold. The energy detection threshold is the more important parameter which decides when to decrement the back-off in the access protocol, thus this parameter must be modified each time the density of nodes increases. However, the implementation of ns-3 is such that any signal below the energy detection threshold is systematically ignored by the node. When the energy detection threshold is high, transmissions from close vehicles may be rejected even if they are successfully transmitted according to the SIR rule.

\section{CONCLUSION}

In this paper, we present a simple stochastic model for spatial CSMA and we compare the results obtained by the model with those obtained by two different simulators: the network simulator ns-3, and a simulator that implements the IEEE 802.11 CSMA/CA protocol using the $\mathrm{C}$ programming language. Overall, the results show a fairly good matching. The increase in the density of successful transmissions when the carrier sense threshold is accurately chosen is well predicted by the model. However, the exact value of the carrier sense threshold required to optimize the throughput is not precisely determined. This is due to the fact that CSMA is difficult to model. The model developed contains many simplifications and approximations and it seems difficult to improve this model. Phenomena such as node starvation, capture of the channel by a group of nodes, etc. lead to a great variation in the simulation results and thus make the analysis much more complex. Nonetheless, the model shows interesting performance prediction capabilities. It also emphasizes the need to optimize the carrier-sense threshold according to variations in node density. Overall, the results show the potential for significant improvements in performance for VANETs and other ad-hoc mobile wireless networks.

\section{REFERENCES}

[1] F. Baccelli, B. Blaszczyszyn, and P. Muhlethaler, "An aloha protocol for multihop mobile wireless networks," Information Theory, IEEE Transactions on, vol. 52, no. 2, pp. 421-436, Feb 2006.

[2] L. Kleinrock and F. Tobagi, "Packet switching in radio channels: Part I-carrier sense multiple-access modes and their throughput-delay characteristics," IEEE Transactions on Communications, vol. COM23, no. 12, pp. 1400-1416, December 1975, (Also, "Multiple Access Communications, Foundations for Emerging Technologies", Norman Abramson (Ed), IEEE Press, 1992, pp. 272-288.).

[3] G. Bianchi, "Performance Analysis of the IEEE 802.11 Distributed Coordination Function," IEEE Journal of Selected Areas in Communications., vol. 18, no. 3, pp. 535-547, March 2000. [Online]. Available: http://dx.doi.org/10.1109/49.840210

[4] S. Ghez, S. Verdu, and S. Schartz, "Stability properties of slotted Aloha with multipacket reception capability," IEEE Trans. Automat. Contr., vol 7, pp. 640-648, 1988.

[5] P. Jacquet and P. Muhlethaler, "Mean number of transmissions with csma in a linear network," in 2010 IEEE 72nd Vehicular Technology Conference: VTC2010-Fall, 69 September 2010, Ottawa, Canada 2010.

[6] P. Muhlethaler and A. Najid, "Throughput optimization in multihop csma mobile ad hoc networks," in EW 2004. The 5th European Wireless Conference, February 24 - 27. Barcelona 2004.

[7] D. Stoyan, W. S. Kendall, and J. Mecke, Stochastic geometry and its applications. 2nd edition. Wiley, 1995.

[8] A. Busson and G. Chelius, "Point processes for interference modeling in csma/ca ad-hoc networks," in Conference: Proceedings of the 6th ACM International Workshop on Performance Evaluation of Wireless Ad Hoc, Sensor, and Ubiquitous Networks, PE-WASUN 2009, October 28-29 2009, Tenerife, Canary Islands, Spain, 2009.

[9] F. Baccelli and B. Błaszczyszyn, Stochastic Geometry and Wireless Networks, Volume II - Applications, ser. Foundations and Trends in Networking. NoW Publishers, 2009, vol. 4, No 1-2.

[10] I. Ramachandran and S. Roy, "Analysis of throughput and energy efficiency of p-persistent csma with imperfect carrier sensing," in GLOBECOM '05. IEEE, 2-4 Dec 2005, St Louis, MO USA 2005.

[11] - "On the impact of clear channel assessment on mac performance," in GLOBECOM '06. IEEE, 27 Nov 1 Dec 2006, San Francisco, California USA 2006.

[12] F. Baccelli and B. Błaszczyszyn, Stochastic Geometry and Wireless Networks, Volume I - Theory, ser. Foundations and Trends in Networking. NoW Publishers, 2009, vol. 3, No 3-4.

[13] M. Lacage and T. R. Henderson, "Yet another network simulator," in Proceeding from the 2006 Workshop on Ns-2: The IP Network Simulator, ser. WNS2 '06. New York, NY, USA: ACM, 2006. [Online]. Available: http://doi.acm.org/10.1145/1190455.1190467 